\title{
Carnets
}

Revue électronique d'études françaises de l'APEF

Deuxième série - 9 | 2017

Reconnaissances et légitimité en français

\section{La traduction, instrument de légitimité littéraire}

Le soutien de l'État français à la traduction au Portugal

Dominique Faria

\section{OpenEdition}

Journals

Édition électronique

URL : http://journals.openedition.org/carnets/2083

DOI : $10.4000 /$ carnets.2083

ISSN : 1646-7698

Éditeur

APEF

Référence électronique

Dominique Faria, «La traduction, instrument de légitimité littéraire », Carnets [En ligne], Deuxième série - 9 | 2017, mis en ligne le 31 janvier 2017, consulté le 19 avril 2019. URL : http://

journals.openedition.org/carnets/2083; DOI : 10.4000/carnets.2083

Ce document a été généré automatiquement le 19 avril 2019

\section{(c) (1) 8}

Carnets est mis à disposition selon les termes de la licence Creative Commons - Atribution - Pas d'utilisation commerciale 4.0 International. 


\title{
La traduction, instrument de légitimité littéraire
}

\author{
Le soutien de l'État français à la traduction au Portugal
}

Dominique Faria

1 La consécration d'un auteur ou la légitimité d'une littérature nationale aux yeux d'autres cultures ne résultent pas seulement de la qualité des textes, des prix littéraires, ou de la tradition culturelle du pays d'origine et son prestige à l'étranger. D'autres facteurs y contribuent, comme la traduction, un agent silencieux dont l'important rôle dans cette dynamique est souvent négligé. À une échelle globale, « les cultures littéraires nationales gagnent du prestige lorsqu'elles sont représentées dans d'autres environnements culturels par leurs auteurs » (Kurschus, $2013: 291)^{1}$. Et elles le sont essentiellement par la traduction.

2 Un des atouts majeurs de la théorie des polysystèmes, établie par Itamar Even-Zohar (2000), est de souligner le rôle que joue la traduction dans la constitution des systèmes littéraires nationaux, notamment dans la formation d'un canon de la littérature traduite à l'intérieur du champ littéraire de chaque pays. Toute culture construit sa propre image des différentes littératures étrangères et cette perception dépend presque exclusivement des ouvrages qui sont traduits dans la langue nationale correspondante. Ce canon littéraire peut d'ailleurs ne pas coïncider avec celui qui prévaut dans le contexte d'origine où des textes n'ayant pas subi de traduction dans la plupart des langues nationales (donc peu connus à l'étranger) peuvent prendre une place centrale dans le polysystème littéraire national.

3 Qui plus est, même lorsque les textes sont traduits, ils sont lus en dehors de leur contexte d'origine, ce qui ne saurait être sans conséquences:

Le fait que les textes circulent sans leur contexte, qu'ils n'emportent pas avec eux le champ de production (...) dont ils sont le produit et que les récepteurs, étant euxmêmes insérés dans un champ de production différent, les réinterprètent en fonction de la structure du champ de réception, est générateur de formidables malentendus. (Bourdieu, $2002: 4$ ) 
non seulement les ouvrages qui constituent un canon littéraire national peuvent différer de ceux qui le composent à l'étranger, mais aussi le lieu que l'on attribue à tel ou tel ouvrage à l'intérieur du canon, ainsi que les interprétations qu'on en fait, peuvent ne pas coïncider avec ceux qui prévalent au sein de la culture qui reçoit la traduction.

5 Lorsqu'on réfléchit sur la traduction en tant qu'instrument de légitimité et de consécration, il est donc important de s'interroger sur les inclusions et les omissions (les textes traduits et ceux qui ne l'ont pas été), sur les responsables de cette sélection, ainsi que sur la place attribuée aux textes dans le polysystème littéraire d'arrivée (par comparaison avec celui dont ils jouissent dans le contexte d'origine).

Pour ce qui est des intervenants dans le processus de sélection des textes, les plus évidents sont les maisons d'édition et les traducteurs eux-mêmes. Dans cet article, je m'intéresserai à un autre genre d'entité, que l'on pourrait inclure dans la catégorie du patronage, dont André Lefevere (Lefevere, 1992 : 15-17) souligne l'important rôle dans la détermination des flux de traduction. Je m'arrêterai plus spécifiquement sur le rôle de l'État français dans la diffusion de la littérature nationale à l'étranger par les stratégies actuelles de promotion du livre, particulièrement celles portant sur la traduction.

Si on envisage, à la suite de Pierre Bourdieu (1992 : 235-236), le champ littéraire comme possédant toujours un pôle de grande production, déterminé par la logique du profit commercial et un pôle de production restreint, une littérature à faible tirage, mais à fort degré de consécration, on comprend que ce genre d'initiatives de la part d'organismes étatiques permet de contrecarrer les pressions des logiques marchandes et de favoriser la traduction des textes appartenant au pôle de production restreinte.

8 Sans ce soutien de l'État, cette littérature restera probablement méconnue dans d'autres pays. Pour illustrer ceci, dans la seconde partie de cet article, je m'interrogerai très brièvement sur la réception du roman français de l'extrême contemporain au Portugal. Je partirai d'un groupe d'onze auteurs envisagés comme produisant une littérature réputée de qualité en France pour vérifier si leurs romans sont traduits au Portugal. Cet exemple permettra de lever un peu le voile sur les différences entre le canon du roman français contemporain tel qu'il est conçu dans ces deux pays.

\section{Le soutien du CNL à la traduction de la littérature française au Portugal}

De nos jours, les mécènes ne sont plus les rois et les membres de l'aristocratie auxquels on dédie la première édition d'un ouvrage, s'efforçant de leur plaire. Les États nationaux sont les nouveaux mécènes, qui s'impliquent dans la promotion de leur littérature à l'étranger. En France, deux organismes sont plus directement chargés de cette tâche, le Centre National du Livre et le Ministère des Affaires Étrangères, à travers les ambassades. Selon Xavier Darcos, cet intérêt de la diplomatie culturelle française s'explique notamment par le fait qu'avec la littérature circulent aussi la langue, les idées et l'imaginaire français (Darcos, 2011: 12), mais aussi parce que «l'industrie du livre représente le deuxième poste d'exportation de la France dans le domaine des biens culturels; près d'un quart du chiffre d'affaires de l'édition française est issu de l'exportation et de la cession de droits à l'international » (idem: 13) Comme le soutient Lefevere (2003 : 2), la traduction est question « d'autorité et de légitimité », plutôt que de 
dictionnaires. L'action concertée de l'État français obéit à des critères économiques aussi bien que politiques.

Le Ministère des Affaires Étrangères qui, selon Danièle Sapiro (2010: 217) a longtemps "privilégié la politique de la protection de la langue française (...) au détriment de la traduction vers d'autres langues ", a mis en place à travers les ambassades et les instituts français, des Programmes d'Aide à la Publication depuis 20 ans. Selon le texte d'introduction sur le site de l'Institut Français, cette mesure a «permis de contribuer à la traduction de près de 20000 titres d'auteurs français dans 80 pays. Ce soutien à l'écrit est l'un des outils majeurs dans le développement de l'influence de la littérature et de la pensée françaises à travers le monde. $»^{2}$ N'ayant pas pu trouver de bilan récent des aides à la traduction de la part de ces institutions, je m'occuperai plus en détail du soutien accordé à la traduction par le Centre National du Livre (CNL), un établissement du Ministère de la Culture et de la Communication.

Le CNL met en place, chaque année, un programme d'Aides à la Traduction. Selon le Bilan des aides 2014, le plus récent publié à ce jour, cette année-là un total de 865679 euros d'aides a été accordé à la traduction d'ouvrages français à l'étranger. La modalité la plus importante de ce soutien consiste dans des subventions qui couvrent entre $30 \%$ et $60 \%$ des frais de traduction, suite à une évaluation des dossiers déposés par les éditeurs étrangers auprès de cet organisme. En 2014, le CNL a attribué des aides à la traduction de 284 ouvrages français à l'étranger, dans 36 pays différents au montant de près de 600000 euros, des chiffres qui montrent bien l'investissement de l'État français dans ce secteur de la diplomatie française.

Pour ce qui est du processus de sélection des ouvrages à traduire, les critères adoptés par le CNL sont publiés sur son site :

- La qualité et l'originalité de l'ouvrage d'origine ;

- L'intérêt de sa traduction;

- La cohérence par rapport à la politique éditoriale et au catalogue de l'éditeur ;

- La qualité de l'échantillon de traduction fourni ;

- La compétence du traducteur ;

- Les difficultés particulières de la traduction;

- Les risques commerciaux pris par l'éditeur et la viabilité économique du projet ;

- Le contexte du pays dans lequel le demandeur exerce son activité3.

13 Certains de ces critères, si on les applique à la littérature, semblent suggérer que le CNL privilégie le soutien à la traduction de textes appartenant au pôle de production restreint : on privilégie les ouvrages dont la traduction pose de difficultés particulières et les projets représentant un plus grand risque commercial pour l'éditeur. Dans l'ensemble, ces critères sont néanmoins assez vagues. Le dernier, par exemple, montre qu'il y a des préférences par rapport aux pays cible sans fournir de critères plus spécifiques ${ }^{4}$.

En analysant les listes publiées par le CNL, on s'aperçoit que les pays auxquels a été attribué le plus grande nombre d'aides - mon critère est le nombre d'ouvrages traduits (littéraires ou appartenant aux sciences humaines et sociales) et pas le montant - sont la Serbie (23 ouvrages), l'Espagne (22 ouvrages), la Grèce (19 ouvrages), les États-Unis (18 ouvrages), l'Italie (16 ouvrages) et le Royaume-Uni (15 ouvrages). Dans cette première liste, les pays anglophones sont un choix évident, de par l'importance de la langue anglaise dans le monde globalisé $e^{5}$ l'Espagne et l'Italie aussi, car ce sont des pays voisins. Les raisons des choix de la Serbie et de la Grèce sont moins évidentes et se doivent 
sûrement à un plan stratégique du CNL. Suivent l'Allemagne et l'Argentine (11 ouvrages), la Russie (10 ouvrages), la Croatie, la Bulgarie, le Danemark et le Portugal (9 ouvrages), l'Albanie (8 ouvrages), Israël et la Turquie (7 ouvrages). Dans les autres vingt pays le CNL a soutenu la traduction de moins de cinq textes par pays. On remarque tout de suite que le Portugal est, de ces seize pays, celui qui a le moins profité des soutiens à la traduction, surtout si on établit une comparaison avec des pays voisins, comme l'Espagne et l'Italie. Nous n'avons évidemment pas les moyens de savoir si cela relève d'une stratégie du CNL ou d'un manque d'intérêt de la part des éditeurs portugais, qui doivent déposer des dossiers pour recevoir l'aide. Ce qui est sûr, c'est que cette distribution asymétrique des fonds a des conséquences sur la quantité et la qualité des traductions d'ouvrages français dans les différents pays. Le graphique ci-dessus rend ceci plus visible : la plupart des aides ont été accordées à cinq pays sur un total de 36.

Soutien à la traduction 2014

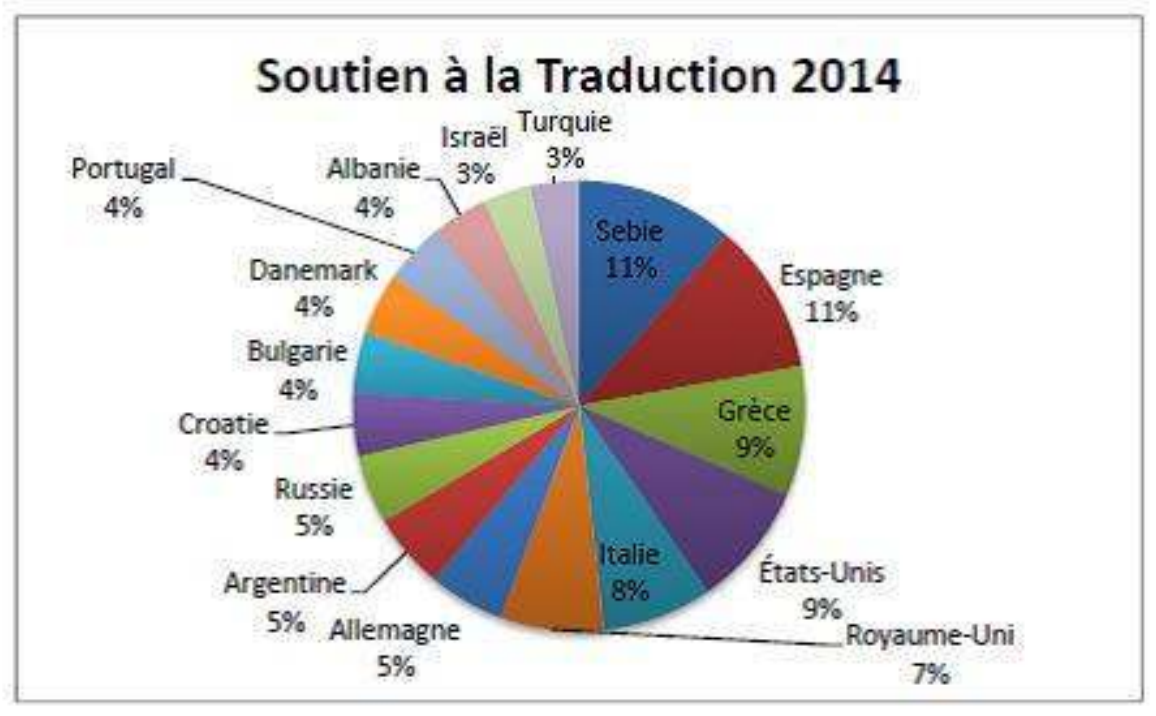

Pour ce qui est des projets retenus pour le Portugal, ils ont été déposés par de petites (et souvent jeunes) maisons d'édition, qui s'adressent à un public spécialisé : Orfeu Negro, créée en 2007, a reçu une aide pour la traduction de trois ouvrages, Antigona, qui date de 1979, pour deux ouvrages, Texto \& Grafia, de 2008, pour 2 ouvrages et Terra Ocre, créée en 1997, pour un ouvrage. Seule Edições 70, qui a traduit un seul livre avec le soutien du $\mathrm{CNL}$, est une maison d'édition établie et possédant un grand nombre de livres dans son catalogue. Le montant total investi au Portugal est de 26125 euros, ce qui correspond à $4 \%$ de l'investissement total et s'est matérialisé dans la traduction de ces neuf ouvrages :

- Marie-José Mondzain, Homo spectator ;

- Henri Behar, Dada : histoire d'une subversion;

- François Truffaut, Les films de ma vie ;

- Jean-Luc Nancy, L'adoration : déconstruction du christianisme II ;

- Pierre-Noël Giraud, La mondialisation, émergences et fragmentations ;

- Pierre Bourdieu, Sur l'Etat. Cours au Collège de France, 1989-1992 ;

- Michel Pastoureau, Noir : histoire d'une couleur ;

- Claude Quetel, Histoire de la folie, de l'Antiquité à nos jours ;

- Jean Meckert, Les coups . 
Un seul de ces textes est fictionnel, le premier roman de l'auteur de romans noirs Jean Mackert. Les autres sont écrits par des philosophes, des historiens, des sociologues, un économiste, un cinéaste, un historien de la littérature. Le soutien du CNL à la traduction au Portugal a donc porté essentiellement sur le domaine des sciences humaines et sociales ${ }^{6}$. Si on compare ces résultats avec ceux des aides attribuées en 2013 et en 2012, on s'aperçoit qu'il s'agit d'une constante. Ainsi, en 2013, huit projets ont obtenu le soutien du $\mathrm{CNL}$, dont 3 sont des romans ${ }^{7}$. En 2012, la traduction de 7 ouvrages a été soutenue, dont un seul est un roman ${ }^{8}$. Dans la liste des maisons d'édition portugaises ayant reçu cette aide en 2012 et 2013, nous retrouvons aussi des constantes. Orféu Negro, Texto \& Grafia et Antigona réapparaissent, côte à côte avec d'autres petites maisons d'éditions : Terreiro do Paço Editores, Santillana Editores et KKYM. Une seule grande maison d'édition surgit sur ces listes, Relógio d'Água. Que ceci se doive à un choix politique de la France ou aux initiatives des maisons d'éditions portugaises, le fait est qu'il n'y a pas depuis 2012 d'aide soutenue à la traduction de littérature française au Portugal de la part du CNL.

\section{Quel canon pour le roman français de l'extrême contemporain au Portugal ?}

17 Pour exemplifier et complémenter la réflexion menée jusqu'ici sur le rôle de la traduction dans la formation des canons littéraires et l'accroissement du capital symbolique d'une culture, je me questionnerai sur la traduction au Portugal des ouvrages de onze romanciers contemporains qui publient chez Minuit (bien que souvent pas en exclusivité) et dont la qualité est reconnue en France. Le choix des Éditions de Minuit s'est imposé dû à sa réputation de publier une littérature de qualité, d'avant-garde (Simonin, 1994 : 471), s'adressant à un public intellectuel (Bourdieu, 1992 : 239-240), appartenant donc au pôle de production restreinte dont on parlait dans l'introduction à cet article.

De ces onze auteurs, cinq n'ont aucun roman traduit au Portugal (voir tableau 1). Ce sont pourtant des noms assez connus en France, des auteurs ayant tous publiés plus d'une dizaine de romans chacun : Eric Chevillard, Yves Ravey, Christian Oster, Éric Laurrent, Patrick Deville.

Tableau 1 : Auteurs n'ayant aucun roman publié au Portugal

\begin{tabular}{|l|l|l|}
\hline Auteur & Nombre de romans publiés en France & Nombre de romans publiés au Portugal \\
\hline Eric Chevillard & 20 & 0 \\
\hline Yves Ravey & 16 & 0 \\
\hline Christian Oster & 15 & 0 \\
\hline Éric Laurrent & 11 & 0 \\
\hline Patrick Deville & 10 & 0 \\
\hline
\end{tabular}

Des six auteurs ayant des romans publiés au Portugal (voir tableau 2), cinq n'ont qu'un seul ouvrage traduit : Antoine Volodine, Marie Ndiaye, Jean Christian Gailly, Jean-Philippe 
Toussaint et Marie Redonnet. L'exception en est Jean Echenoz qui a vu cinq de ses seize romans traduits en portugais.

Tableau 2 : Auteurs traduits au Portugal

\begin{tabular}{|l|l|l|}
\hline Auteur & $\begin{array}{l}\text { Nombre de romans publiés en } \\
\text { France }\end{array}$ & $\begin{array}{l}\text { Nombre de romans publiés au } \\
\text { Portugal }\end{array}$ \\
\hline Antoine Volodine & 18 & 1 \\
\hline Marie Ndiaye & 18 & 1 \\
\hline Jean Echenoz & 16 & 5 \\
\hline Christian Gailly & 14 & 1 \\
\hline Jean-Philippe & 13 & 1 \\
Toussaint & 7 & 1 \\
\hline Marie Redonnet & & \\
\hline
\end{tabular}

Les études sur la traduction s'occupent souvent à signaler des écarts du texte traduit par rapport au texte de départ. Ici, ce qui m'intéresse est plutôt les conséquences de la nontraduction. Si on pense que la majorité des lecteurs portugais ne lit pas de textes en français, cette liste montre qu'il y a plusieurs auteurs d'une littérature réputée de qualité qui - malgré le grand nombre d'ouvrages publiés, de prix décernés, de recherche suscitée par leur travail, de traductions dans d'autres langues, dont des langues centrales comme l'anglais - sont méconnus au Portugal.

21 En revanche, d'autres romanciers français sont systématiquement traduits au Portugal. C'est de cas d'olivier Rolin, qui publie chez Seuil, et dont les onze romans sont tous traduits, et de Michel Houellebecq, qui a publié 6 romans dont un seul (paru en 2015) n'est pas encore traduit ${ }^{9}$. Ceci voudrait dire que ces auteurs qui publient aux éditions de Minuit, sauf peut-être Jean Echenoz, ne font pas partie du canon du roman français contemporain au Portugal, alors que d'autres romanciers, comme Olivier Rolin et Michel Houellebecq sont plus facilement reconnus par les lecteurs portugais.

22 Jean Echenoz étant le seul de ces romanciers publiant chez Minuit qui peut être connu des lecteurs portugais, je m'arrêterai brièvement sur quelques aspects de sa traduction au Portugal. En France, il s'est vu décerner de nombreux prix littéraires (notamment le Goncourt), il a intégré depuis longtemps le corpus scolaire. Il s'agit d'un auteur traduit dans une quinzaine de langues. Aux États-Unis, par exemple, des seize livres publiés par Echenoz, treize ont déjà été traduits. Qui plus est, deux traducteurs (renommés) se sont spécialisés dans la traduction de ses textes, Mark Polizzotti et, plus récemment, Linda Coverdale.

Au Portugal, en revanche, seuls 6 ouvrages - dont 5 romans - ont été traduits. Ils l'ont été par six traducteurs différents (Manuela Torres, Manuel Reis, Lurdes Júdice, Armando Silva Carvalho et Virgílio Tenreiro), chez quatre petites maisons d'édition différentes (Terramar, Ambar, Sudoeste, Cavalo de Ferro), plus ou moins méconnues au Portugal. Il n'y a donc pas de projet éditorial autour du travail de cet auteur. Ses textes subissent au 
Portugal ce que Bourdieu appelle une "opération de marquage ", "à travers la maison d'édition, la collection, le traducteur et le préfacier» (Bourdieur, 2002: 4). Il y a effectivement une différence symbolique et un marquage du texte, lorsque le texte passe des Éditions de Minuit, une des plus prestigieuses maisons d'édition françaises, aux toutes petites maisons d'édition portugaises, qui conditionne la façon dont les textes seront perçus par le lecteur portugais.

Comme conséquence de tout ceci, malgré les six ouvrages traduits, Jean Echenoz est très peu connu au Portugal. Pour exemplifier ceci, je cite la première phrase d'un article de l'hebdomadaire portugais Expresso, qui affirme, à propos de la parution de la traduction portugaise de Courir, en 2011 :

Oubliez le médiatique Michel Houellebecq et croyez-moi : le discret Jean Echenoz, lui il est vraiment un des meilleurs écrivains français de l'actualité. Peu connu parmi nous (malgré quatre livres traduits (...), ce serait une injustice de ne pas s'arrêter sur courir, un roman qui réinvente la vie d'Emil Zapotek ${ }^{10}$ (Leonardo, 2011 : s.p.)

Cette affirmation nous intéresse car elle sous-entend que, pour le lecteur portugais, le roman français contemporain c'est Houellebecq, peut-être car il est médiatique et controversé, mais surtout car il est systématiquement traduit en portugais.

\section{Conclusion}

La légitimité et la consécration d'un auteur, d'une littérature nationale dépendent de nombreux facteurs, un des plus importants étant la traduction. Dans un monde globalisé, où l'anglais est hégémonique, le programme de soutien à l'extraduction - la traduction du français vers des langues étrangères - mis en place par l'état français, a le pouvoir de contrer les enjeux éditoriaux et les logiques commerciales et de stimuler la traduction de la littérature réputée de qualité, qui autrement aurait moins de chances d'être traduite et donc connue à l'étranger.

L'analyse des aides à la traduction d'ouvrages français au Portugal attribuées par le CNL entre 2012 et 2014 montre que ce soutien couvre essentiellement des livres appartenant au domaine des sciences humaines et sociales, étant rarement attribué à des textes fictionnels. Il n'est donc pas surprenant qu'un grand nombre de romanciers français ne soient pas traduits au Portugal et que le canon du roman français de l'extrême contemporain tel qu'il est conçu dans ce pays soit assez différent de celui qui prévaut en France.

\section{BIBLIOGRAPHIE}

BOURDIEU, Pierre (1992). Les Règles de l'art. Paris : Seuil.

BOURDIEU, Pierre (2002). «Les conditions sociales de la circulation internationale des idées », in

Actes de la recherche en sciences sociales, vol. 145, décembre 2002, pp. 3-8. 
DARCos, Xavier (2011). « Au service du livre et de l'écrit. Nouvelles missions de l'Institut français ", in Dominique Viart (éd.). La littérature française du $20^{e}$ siècle lue de l'étranger. Villeneuve d'Ascq : Presses Universitaires du Septentrion.

EVEN-ZOHAR, Itamar (2000). "The position of translated literature within the literary polysystem » , in Lawrence Venuti (ed.). The translation studies reader. London, New York: Routledge, pp. 192-197.

KURSCHUS, Stéphanie (2013). European book cultures. Diversity as a challenge. Weisbaden: Springer VS.

LEFEVERE, André (1992). Translation, Rewriting and the Manipulation of Literary Fame, London, New York: Routledge.

LEFEVERE, André (2003). « Introduction », in André Lefevere (ed.). Translation, history, culture. Londres, New York : Routledge, pp. 1-13.

LEONARDO, Ana Cristina (2011). « Correr », Expresso, 16-04-2011.

SAPIRO, Gisèle (2010). « Les échanges culturels entre la France et Israël », in Anne Dulphy et al, (dir.). Les relations culturelles internationales au $\mathrm{xx}^{e}$ siècle : de la diplomatie culturelle à l'acculturation. Bruxelles : Peter Lang.

SIMONIN, Anne (1994). Les Editions de minuit 1942-1955, le devoir d'insoumission. Paris : IMEC.

\section{NOTES}

1. Je traduis.

2. Voir <URL: http://www.institutfrancais.com/fr/actualit\%C3\%A9s/programmes-daide-lapublication>

3. Voir :<URL: http://www.centrenationaldulivre.fr/fr/editeur/aide_a_la_traduction/ aide_pour_la_traduction_d_ouvrages_francais_en_langues_etrangeres/>

4. Les préférences changent d'une année à l'autre, selon les priorités établies en accord avec les spécificités des relations diplomatiques entre la France et d'autres pays. En 2014, par exemple, la célébration des 50 ans de relations diplomatiques entre la France et la Chine expliquent l'accord d'un plus grand soutien à la traduction dans ce pays.

5. Où être traduit en anglais implique également une possibilité d'être traduit dans des langues plus exotiques, par le biais de la traduction indirecte.

6. Le CNL attribue également des bourses destinées aux traducteurs étrangers, qui ont déjà un contrat avec un éditeur, pour des séjours en France, destinés à permettre de mener un projet de traduction sur place. En 2014, 89 traducteurs ont reçu cette bourse, au montant de 272000 euros. Si on analyse les listes fournies par le $\mathrm{CNL}$, on s'aperçoit que les provenances des traducteurs couvrent 31 pays différents, ceux qui ont le plus bénéficié de cette aide étant : la Chine (avec 15 traducteurs), le Vietnam (12), la Roumanie et la Turquie (7), la Pologne et la Géorgie (5), la Croatie (3), la Rép. Tchèque, l'Arménie, l'Argentine, l'Iran, la Slovénie, la Serbie, l'Ukraine, la Lituanie (2). Pour les autres pays, un seul traducteur a reçu cette subvention. Aucun traducteur du Portugal n'a joui de ces bourses.

7. Joël Dicker, La vérité sur l'affaire Harry Quebert (roman); Jean-Baptiste Duroselle, Histoire des relations internationales, 1919 à 1945 ; Jean-Baptiste Duroselle, Histoire des relations internationales, de 1945 à nos jours ; Jacques Ellul, Propagandes ; Jérôme Ferrari, Le sermon sur la chute de Rome (roman) ; Michel Houellebecq, Les particules élémentaires (roman); Jacques Rancière, La fable cinématographique ; Jacques Rancière, Bela TARR. 
8. Cathy Bernheim, Mary Shelley - La jeune fille et le monstre; Pierre Bourdieu, La domination masculine; Christian Delacampagne, Histoire de l'esclavage; Georges Didihuberman, Atlas ou le gai savoir inquiet; Eric Dufour, Le cinéma de science-fiction; Jean Malaquais, Les Javanais (roman); Jacques Rancière, Les écarts du cinéma.

9. Notons qu'au moins deux romans de cet auteur ont été traduits au Portugal avec le soutien du CNL (La Carte et le territoire et Les Particules élémentaires).

10. Je traduis.

\section{RÉSUMÉS}

La traduction joue un rôle essentiel dans l'accroissement du capital symbolique des littératures nationales. Dans cet article, je me questionne sur les mesures adoptées par l'État français dans le soutien à la traduction, à travers les programmes comme celui du CNL, notamment au Portugal. Cette intervention étatique a d'importantes conséquences dans la promotion d'une littérature de qualité, peu commerciale, qui autrement risque d'être méconnue à l'étranger.

Translation plays a crucial role in increasing the legitimacy of national literatures. In this paper I underline the role played by the French State in sustaining translation, through programs such as that of $\mathrm{CNL}$, namely in Portugal. Such governmental measures have important consequences on the promotion of quality literature which, because it is not very commercial, tends to be less known abroad.

\section{INDEX}

Mots-clés : traduction, légitimité, littérature française, Portugal, roman français contemporain Keywords : translation, legitimacy, French literature, Portugal, French contemporary novel

\section{AUTEUR}

\section{DOMINIQUE FARIA}

Un. des Açores/CEC-Lisboa

dominique.ar.faria[at]uac.pt 